\title{
Supplemental Materials for: Longitudinal Mixed Membership Trajectory Models for Disability Survey Data
}

Daniel Manrique-Vallier*

July 29,2014

\section{TGoM model with piece-wise constant trajectories}

The specific choice extreme trajectories in the TGoM model is arbitrary. In our application to the NLTCS we have specified simple sigmoid curves, based on the expectation of monotonicity of the probability of disability as a function of age. This specification produced sound and interpretable estimates (up to $K=4$ ). In this complementary section we investigate the effects of this assumption by replacing the extreme trajectories specification, $\lambda_{j k}(\tau)$, with a less constrained specification.

For this investigation we use piece-wise constant functions. Consider the partition of the range of possible birth dates into $c$ contiguous intervals, $B_{1}=\left(-\infty, c_{1}\right], B_{2}=\left(c_{1}, c_{2}\right], \ldots, B_{c}=$ $\left(c_{c}, \infty\right)$, where $c_{1}<c_{2}<\ldots<c_{c-1}$ are arbitrary breakpoints. Call $\mathcal{B}=\left\{B_{1}, B_{2} \ldots, B_{c}\right\}$. We

${ }^{*}$ Department of Statistics, Indiana University. Email:dmanriqu@indiana.edu 
define the piecewise-constant trajectory function, $\lambda_{j k}(\tau)$, as

$$
\lambda_{j t}(\tau)=\prod_{b \in \mathcal{B}}\left[\lambda_{(b) j k}\right]^{I(\tau \in b)}
$$

where $0<\lambda_{(b) j k}<1$ are constants for each combination of $b \in \mathcal{B}, j, k$. We replace this specification into the definition of the TGoM model. The idea is to provide an easy to estimate, even if crude, form of non-parametric curve estimation.

For our application, we define seven breakpoints, $c_{1}=68, c_{2}=71, c_{3}=74, c_{4}=77, c_{5}=$ $80, c_{6}=83$ and $c_{7}=86$. This particular choice defines trajectories over time with 8 steps per combination of ADL and extreme profile. It therefore requires $8 \times J \times K$ parameters. We assume a prior distribution $\lambda_{j k b} \stackrel{i i d}{\sim} \operatorname{Beta}(1,1)$ for all $j, k$ and $b$. For $\boldsymbol{\alpha}$ we assume the same prior specification that we used for the basic TGoM with continuous trajectories: $\alpha_{0} \sim \operatorname{Gamma}(1,5), \boldsymbol{\xi} \sim \operatorname{Dirichlet}\left(\mathbf{1}_{K}\right)$ and $\boldsymbol{\alpha}=\left(\alpha_{0} \cdot \xi_{1}, \ldots, \alpha_{0} \cdot \xi_{K}\right)$.

Fitting the model using MCMC sampling we get posterior estimates for $\alpha_{0}$ and $\xi$ that are almost identical to those obtained through the application of the basic model with continuous trajectories (see Table 1).

Figures 1, 2 and 3 show estimated posterior extreme trajectories for models with $K=$ 3,4 and 5, overlaid with the continuous trajectories estimated from the Basic Model with continuous trajectories for comparison. We see that the discrete and continuous trajectories for the most important extreme profiles $(k=1,2)$ are essentially the same in all cases. Extreme profile $k=3$ in models with $K=3$ (Figure 1, last row) and extreme profile $k=4$ in models with $K=4$ (Figure 2, last row) diverge when approaching late ages. We observe this behavior at late ages in profiles where subjects have an increased probability of acquiring disabilities early in life. A likely explanation is that subjects for whom those characteristics are predominant, tend to die earlier than their peers that are closer to healthier profiles. Looking at profile $k=5$ for models with $K=5$ extreme profiles (Figure 3) we see that all 


\begin{tabular}{|l|c|l|}
\hline Model & Parameter & Estimate [s.d.] \\
\hline \hline \multirow{2}{*}{$K=2$} & $\alpha_{0}$ & $0.325[0.007]$ \\
& $\xi$ & $(0.820[0.002], 0.180[0.002])$ \\
\hline \multirow{2}{*}{$K=3$} & $\alpha_{0}$ & $0.286[0.006]$ \\
& $\xi$ & $(0.632[0.004], 0.263[0.003], 0.105[0.002])$ \\
\hline \multirow{2}{*}{$K=4$} & $\alpha_{0}$ & $0.269[0.006]$ \\
& $\xi$ & $(0.558[0.004], 0.256[0.004], 0.117[0.003], 0.069[0.001])$ \\
\hline \multirow{2}{*}{$K=5$} & $\alpha_{0}$ & $0.261[0.006]$ \\
& $\xi$ & $(0.519[0.005], 0.251[0.005], 0.130[0.003], 0.050[0.001], 0.050[0.002])$ \\
\hline
\end{tabular}

Table 1: Posterior means for parameters $\alpha_{0}$ and $\xi$ for basic model with discrete step trajectories for $K=2,3,4,5$ extreme profiles. Numbers between brackets are posterior standard deviations.

trajectories behave erratically. This situation mirrors that encountered in the application of the model with continuous trajectories in (overlaid on the same figure).

This analysis shows two things. First, trajectories of the main tendencies in the population do tend to be monotonically increasing. Moreover, the cases where these tendencies do not hold well seem to be due to data sparsity - advanced ages in low-populated profiles with high-mortality rates. This provides empirical justification for the use of parametric specifications that enforce this characteristic, like linear logits, as a way of saving inferential power for extrapolation at advanced ages. Second, the fact that trajectories in profile in the least important profiles of model with $K=5$ behave erratically, while at the same time models with parametric monotonic curves exhibit unsound decreasing tendencies, suggests that the residual variability captured by this extreme profile - even though small —is not well represented as trajectories. Accounting for this variability is an area for future improvements. 


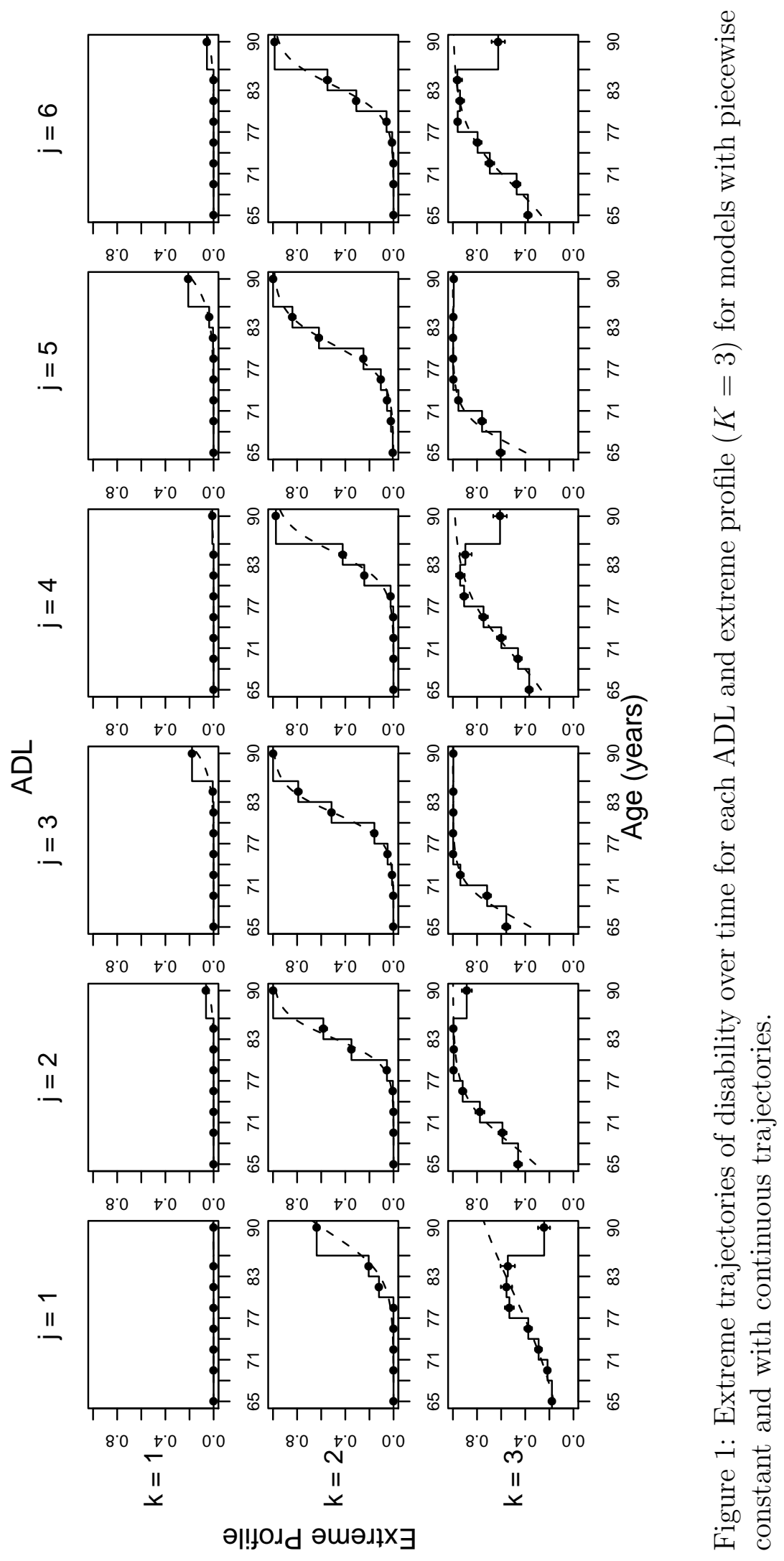




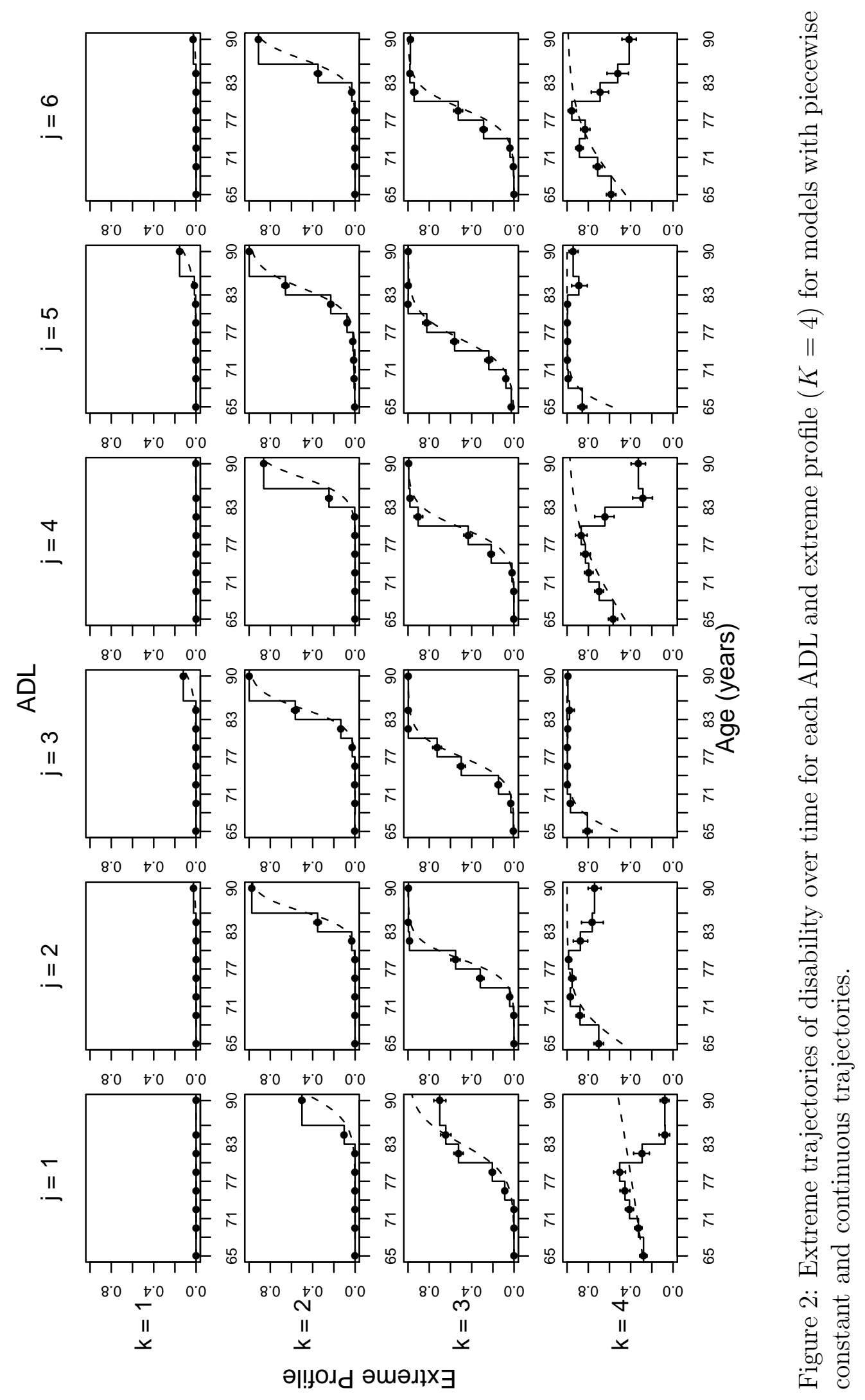




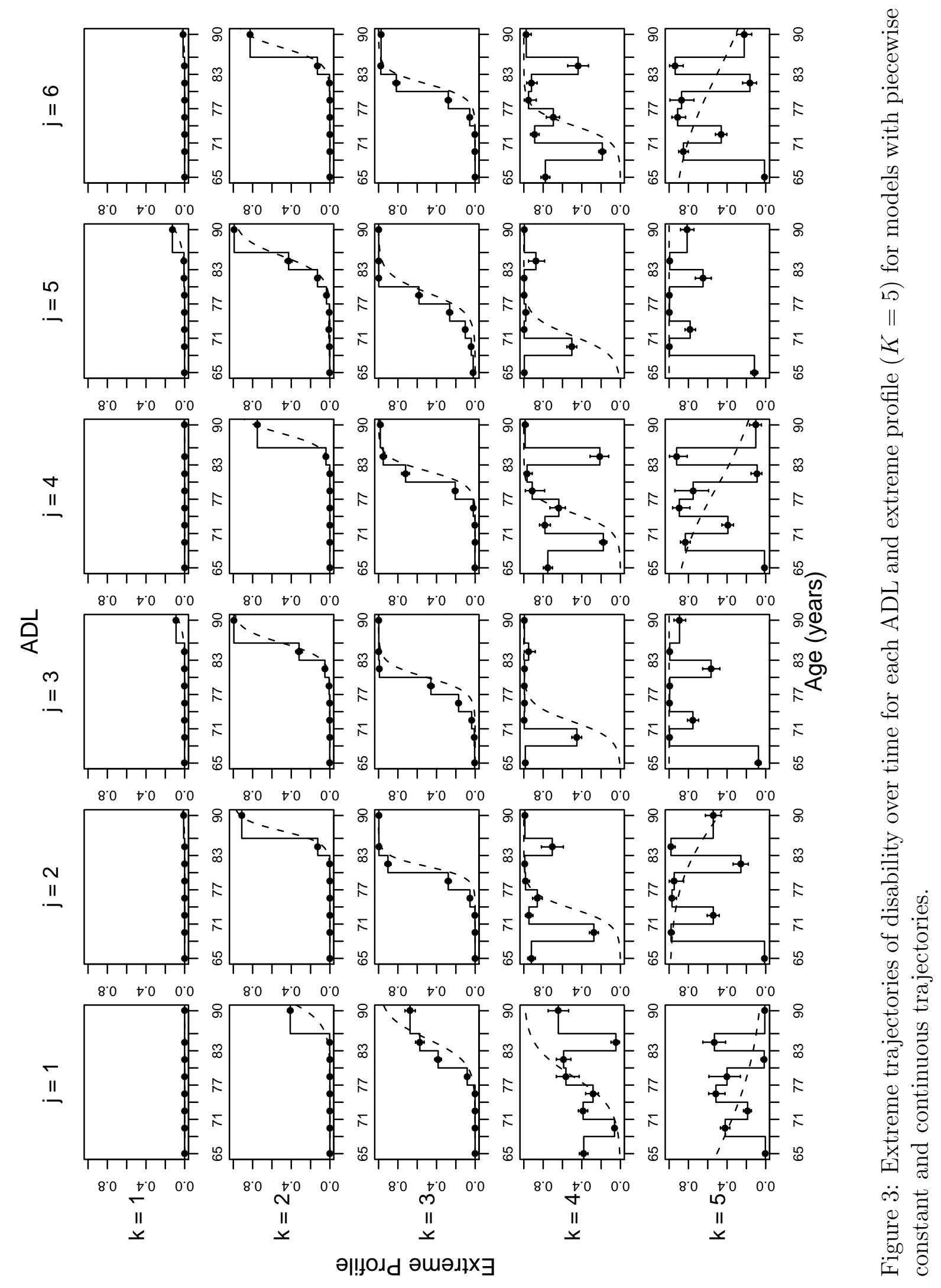




\section{Posterior Estimates for TGoM models}

\begin{tabular}{|c|c|c|c|c|c|}
\hline Parameter & Estimate & & & & \\
\hline$\alpha_{0}$ & $0.328(0.007)$ & & & & \\
\hline \multirow{2}{*}{ Parameter } & \multirow{2}{*}{$\operatorname{ADL}(j)$} & \multicolumn{4}{|c|}{ Estimate Extreme Profile- $k$ (sd) } \\
\hline & & \multicolumn{2}{|c|}{$k=1$} & \multicolumn{2}{|c|}{$k=2$} \\
\hline$\xi$ & - & 0.824 & \begin{tabular}{l|l}
$(0.002)$ \\
\end{tabular} & 0.176 & $(0.002)$ \\
\hline \multirow{6}{*}{$\beta_{0 * *}$} & 1 (EAT) & -6.496 & $(0.121)$ & -0.494 & $(0.026)$ \\
\hline & 2 (BED) & -4.861 & $(0.063)$ & 1.736 & $(0.053)$ \\
\hline & 3 (MOB) & -3.789 & $(0.042)$ & 2.843 & $(0.084)$ \\
\hline & 4 (DRS) & -5.518 & $(0.082)$ & 0.945 & $(0.039)$ \\
\hline & $5(\mathrm{BTH})$ & -3.163 & $(0.032)$ & 3.298 & $(0.097)$ \\
\hline & 6 (TLT) & -4.710 & $(0.059)$ & 1.292 & $(0.045)$ \\
\hline \multirow{6}{*}{$\beta_{1 * *}$} & 1 (EAT) & 0.293 & $(0.008)$ & 0.136 & $(0.003)$ \\
\hline & 2 (BED) & 0.312 & $(0.005)$ & 0.218 & $(0.005)$ \\
\hline & 3 (MOB) & 0.289 & $(0.004)$ & 0.273 & $(0.007)$ \\
\hline & 4 (DRS) & 0.310 & $(0.006)$ & 0.182 & $(0.004)$ \\
\hline & $5(\mathrm{BTH})$ & 0.247 & $(0.003)$ & 0.295 & $(0.008)$ \\
\hline & 6 (TLT) & 0.280 & $(0.005)$ & 0.204 & $(0.005)$ \\
\hline \multirow{6}{*}{$\mathrm{Age}_{1 / 2}$} & 1 (EAT) & 102.171 & $(0.294)$ & 83.641 & $(0.236)$ \\
\hline & 2 (BED) & 95.577 & $(0.130)$ & 72.038 & $(0.144)$ \\
\hline & $3(\mathrm{MOB})$ & 93.128 & $(0.107)$ & 69.589 & $(0.132)$ \\
\hline & $4(\mathrm{DRS})$ & 97.818 & $(0.173)$ & 74.824 & $(0.155)$ \\
\hline & $5(\mathrm{BTH})$ & 92.823 & $(0.120)$ & 68.810 & $(0.124)$ \\
\hline & 6 (TLT) & 96.831 & $(0.160)$ & 73.663 & $(0.144)$ \\
\hline
\end{tabular}

Table 2: Posterior means for parameters of interest for basic TGoM model with $K=2$ extreme profiles. Numbers between parenthesis are posterior standard deviations. 


\begin{tabular}{|c|l|}
\hline Parameter & Estimate \\
\hline$\alpha_{0}$ & $0.261(0.006)$ \\
\hline
\end{tabular}

\begin{tabular}{|c|l|rr|rr|rr|}
\hline \multirow{2}{*}{ Parameter } & \multirow{2}{*}{ ADL $(j)$} & \multicolumn{6}{|c|}{ Estimate Extreme Profile- $k$ (sd) } \\
& & \multicolumn{2}{|c|}{$k=1$} & \multicolumn{2}{c|}{$k=2$} & \multicolumn{2}{c|}{$k=3$} \\
\hline$\xi$ & - & 0.645 & $(0.004)$ & 0.251 & $(0.004)$ & 0.104 & $(0.002)$ \\
\hline \multirow{5}{*}{$\beta_{0 * *}$} & $1(\mathrm{EAT})$ & -8.843 & $(0.322)$ & -3.100 & $(0.060)$ & -0.074 & $(0.046)$ \\
& $2(\mathrm{BED})$ & -7.022 & $(0.151)$ & -1.737 & $(0.055)$ & 3.559 & $(0.151)$ \\
& $3(\mathrm{MOB})$ & -5.332 & $(0.096)$ & -0.757 & $(0.046)$ & 5.734 & $(0.274)$ \\
& $4(\mathrm{DRS})$ & -7.885 & $(0.218)$ & -2.249 & $(0.055)$ & 2.028 & $(0.084)$ \\
& $5(\mathrm{BTH})$ & -4.452 & $(0.071)$ & -0.229 & $(0.039)$ & 6.210 & $(0.304)$ \\
& $6(\mathrm{TLT})$ & -6.587 & $(0.145)$ & -1.768 & $(0.049)$ & 2.491 & $(0.100)$ \\
\hline \multirow{5}{*}{$\beta_{1 * *}$} & $1(\mathrm{EAT})$ & 0.357 & $(0.017)$ & 0.347 & $(0.009)$ & 0.104 & $(0.006)$ \\
& $2(\mathrm{BED})$ & 0.394 & $(0.010)$ & 0.551 & $(0.013)$ & 0.289 & $(0.013)$ \\
& $3(\mathrm{MOB})$ & 0.348 & $(0.007)$ & 0.520 & $(0.012)$ & 0.422 & $(0.022)$ \\
& $4(\mathrm{DRS})$ & 0.390 & $(0.013)$ & 0.462 & $(0.011)$ & 0.202 & $(0.008)$ \\
& $5(\mathrm{BTH})$ & 0.295 & $(0.006)$ & 0.425 & $(0.009)$ & 0.442 & $(0.024)$ \\
& $6(\mathrm{TLT})$ & 0.337 & $(0.009)$ & 0.474 & $(0.011)$ & 0.233 & $(0.009)$ \\
\hline \multirow{5}{*}{ Age $_{1 / 2}$} & $1(\mathrm{EAT})$ & 104.768 & $(0.462)$ & 88.933 & $(0.172)$ & 80.725 & $(0.477)$ \\
& $2(\mathrm{BED})$ & 97.824 & $(0.174)$ & 83.156 & $(0.091)$ & 67.674 & $(0.179)$ \\
& $3(\mathrm{MOB})$ & 95.332 & $(0.139)$ & 81.457 & $(0.084)$ & 66.399 & $(0.155)$ \\
& $4(\mathrm{DRS})$ & 100.210 & $(0.235)$ & 84.873 & $(0.105)$ & 69.959 & $(0.197)$ \\
& $5(\mathrm{BTH})$ & 95.107 & $(0.155)$ & 80.539 & $(0.093)$ & 65.940 & $(0.164)$ \\
& $6(\mathrm{TLT})$ & 99.538 & $(0.231)$ & 83.731 & $(0.097)$ & 69.315 & $(0.181)$ \\
\hline
\end{tabular}

Table 3: Posterior means for parameters of interest for basic TGoM model with $K=3$ extreme profiles. Numbers between parenthesis are posterior standard deviations. 


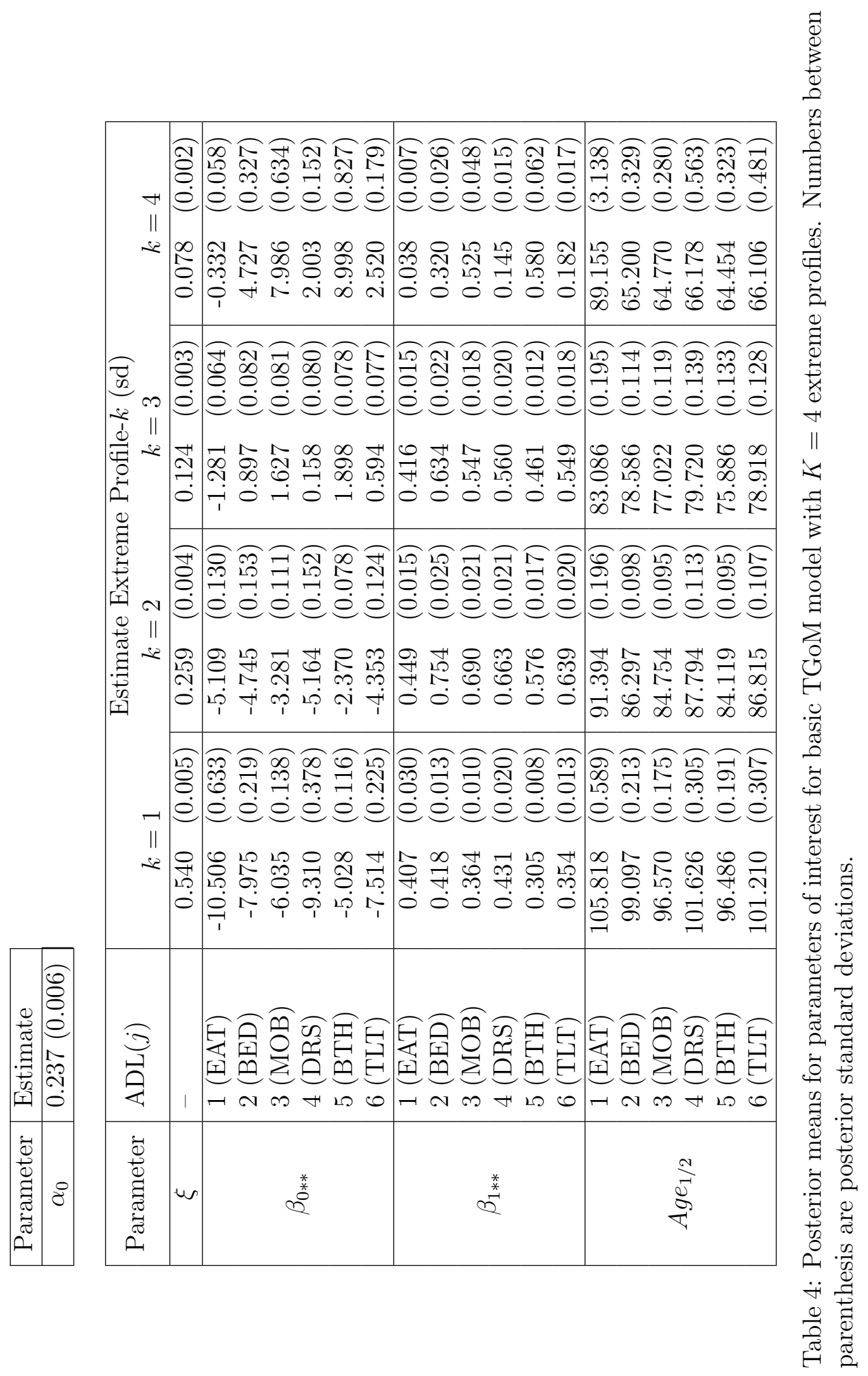




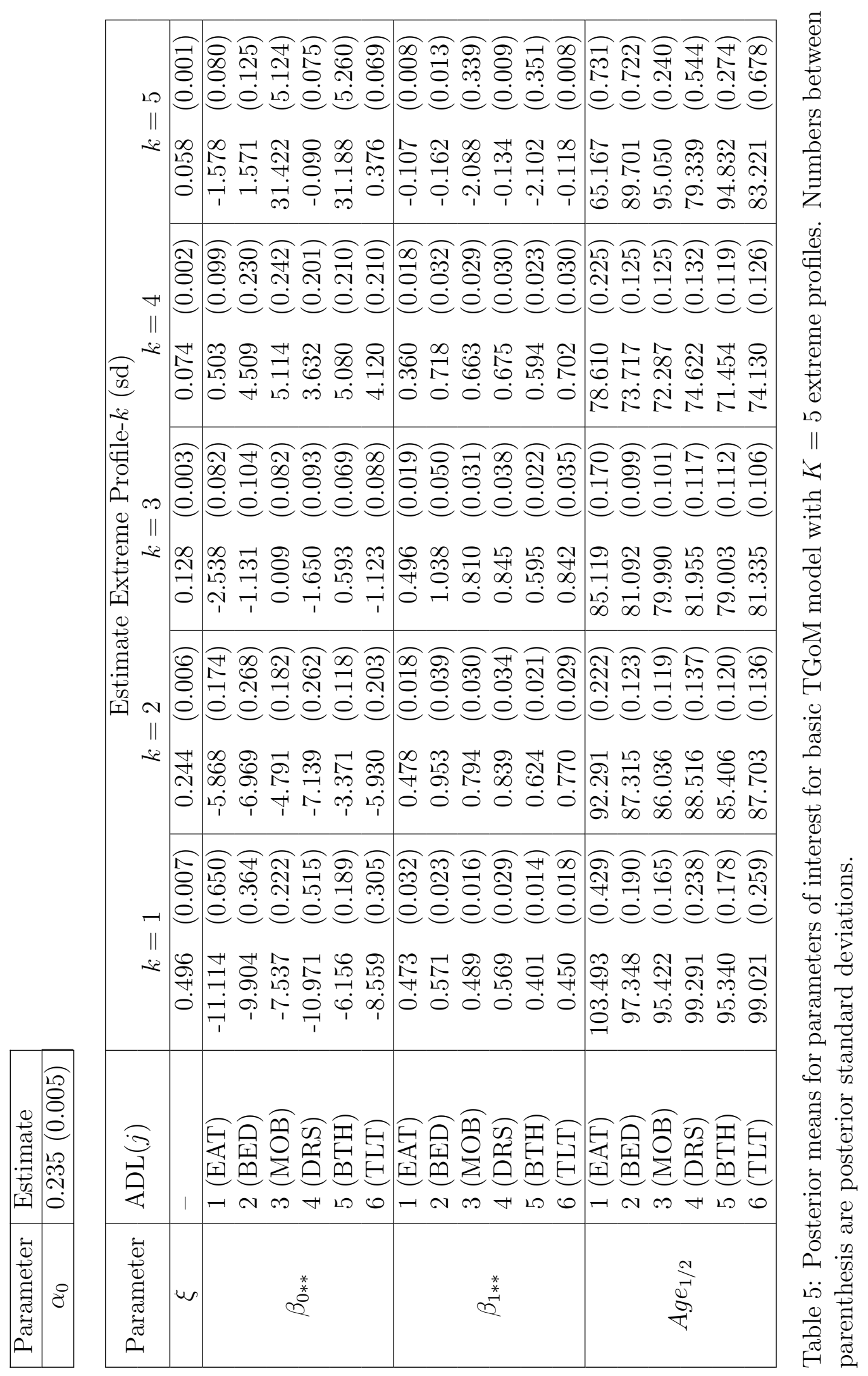




\section{Posterior Estimates for TGoM models with cohort}

\section{extensions}

\begin{tabular}{|c|l|}
\hline Parameter & Estimate \\
\hline$\alpha_{0(5)}$ & $0.137(0.013)$ \\
$\alpha_{0(4)}$ & $0.351(0.016)$ \\
$\alpha_{0(3)}$ & $0.382(0.015)$ \\
$\alpha_{0(2)}$ & $0.412(0.015)$ \\
$\alpha_{0(1)}$ & $0.327(0.011)$ \\
\hline
\end{tabular}

\begin{tabular}{|c|c|c|c|c|c|}
\hline \multirow{2}{*}{ Parameter } & \multirow{2}{*}{ Cohort } & \multicolumn{4}{|c|}{ Estimate Extreme Profile- $k$ (sd) } \\
\hline & & \multicolumn{2}{|c|}{$k=1$} & \multicolumn{2}{|c|}{$k=2$} \\
\hline \multirow{5}{*}{$\xi$} & 5 & 0.908 & $(0.003)$ & 0.092 & $(0.003)$ \\
\hline & 4 & 0.864 & $(0.004)$ & 0.136 & $(0.004)$ \\
\hline & 3 & 0.827 & $(0.004)$ & 0.173 & $(0.004)$ \\
\hline & 2 & 0.804 & $(0.004)$ & 0.196 & $(0.004)$ \\
\hline & 1 & 0.727 & $(0.005)$ & 0.273 & $(0.005)$ \\
\hline \multirow{2}{*}{ Parameter } & \multirow{2}{*}{$\operatorname{ADL}(j)$} & \multirow{2}{*}{\multicolumn{4}{|c|}{$\begin{array}{c}\text { Estimate Extreme Profile- } k(\mathrm{sd}) \\
k=1 \\
k=2\end{array}$}} \\
\hline & & & & & \\
\hline \multirow{6}{*}{$\beta_{0 * *}$} & 1 (EAT) & -6.650 & $(0.132)$ & -0.518 & $(0.025)$ \\
\hline & $2(\mathrm{BED})$ & -4.952 & $(0.068)$ & 1.707 & $(0.052)$ \\
\hline & $3(\mathrm{MOB})$ & -3.848 & $(0.044)$ & 2.840 & $(0.087)$ \\
\hline & $4(\mathrm{DRS})$ & -5.663 & $(0.091)$ & 0.920 & $(0.038)$ \\
\hline & $5(\mathrm{BTH})$ & -3.183 & $(0.033)$ & 3.294 & $(0.101)$ \\
\hline & 6 (TLT) & -4.764 & $(0.062)$ & 1.257 & $(0.044)$ \\
\hline \multirow{6}{*}{$\beta_{1 * *}$} & $1(\mathrm{EAT})$ & 0.293 & $(0.008)$ & 0.130 & $(0.003)$ \\
\hline & $2(\mathrm{BED})$ & 0.312 & $(0.005)$ & 0.211 & $(0.005)$ \\
\hline & $3(\mathrm{MOB})$ & 0.288 & $(0.004)$ & 0.268 & $(0.008)$ \\
\hline & $4(\mathrm{DRS})$ & 0.310 & $(0.007)$ & 0.177 & $(0.004)$ \\
\hline & $5(\mathrm{BTH})$ & 0.243 & $(0.003)$ & 0.290 & $(0.009)$ \\
\hline & 6 (TLT) & 0.276 & $(0.005)$ & 0.196 & $(0.005)$ \\
\hline \multirow{6}{*}{$\mathrm{Age}_{1 / 2}$} & 1 (EAT) & 102.678 & $(0.329)$ & 83.993 & $(0.239)$ \\
\hline & 2 (BED) & 95.898 & $(0.141)$ & 71.912 & $(0.148)$ \\
\hline & 3 (MOB) & 93.382 & $(0.114)$ & 69.398 & $(0.137)$ \\
\hline & $4(\mathrm{DRS})$ & 98.249 & $(0.188)$ & 74.791 & $(0.156)$ \\
\hline & $5(\mathrm{BTH})$ & 93.110 & $(0.123)$ & 68.624 & (0.130) \\
\hline & 6 (TLT) & 97.237 & $(0.174)$ & 73.605 & $(0.146)$ \\
\hline
\end{tabular}

Table 6: Posterior means for parameters of interest for basic model with $K=2$ extreme profiles. Numbers between parenthesis are posterior standard deviations. 


\begin{tabular}{|c|l|}
\hline Parameter & Estimate \\
\hline$\alpha_{0(5)}$ & $0.253(0.019)$ \\
$\alpha_{0(4)}$ & $0.194(0.012)$ \\
$\alpha_{0(3)}$ & $0.224(0.010)$ \\
$\alpha_{0(2)}$ & $0.310(0.011)$ \\
$\alpha_{0(1)}$ & $0.291(0.010)$ \\
\hline
\end{tabular}

\begin{tabular}{|c|c|c|c|c|c|c|c|}
\hline \multirow{2}{*}{ Parameter } & \multirow{2}{*}{ Cohort } & \multicolumn{6}{|c|}{ Estimate Extreme Profile- $k$ (sd) } \\
\hline & & \multicolumn{2}{|c|}{$k=1$} & \multicolumn{2}{|c|}{$k=2$} & \multicolumn{2}{|c|}{$k=3$} \\
\hline \multirow{5}{*}{$\xi$} & 5 & 0.740 & $(0.021)$ & 0.190 & $(0.021)$ & 0.070 & $(0.003)$ \\
\hline & 4 & 0.722 & $(0.007)$ & 0.188 & $(0.007)$ & 0.090 & $(0.003)$ \\
\hline & 3 & 0.642 & $(0.007)$ & 0.250 & $(0.007)$ & 0.108 & $(0.003)$ \\
\hline & 2 & 0.595 & $(0.007)$ & 0.279 & $(0.007)$ & 0.126 & $(0.003)$ \\
\hline & 1 & 0.615 & $(0.006)$ & 0.251 & $(0.006)$ & 0.134 & $(0.005)$ \\
\hline \multirow{2}{*}{ Parameter } & \multirow{2}{*}{$\operatorname{ADL}(j)$} & \multicolumn{6}{|c|}{ Estimate Extreme Profile- $k(\mathrm{sd})$} \\
\hline & & \multicolumn{2}{|c|}{$k=1$} & $k$ & $=2$ & \multicolumn{2}{|c|}{$k=3$} \\
\hline \multirow{6}{*}{$\beta_{0 * *}$} & 1 (EAT) & -9.038 & $(0.364)$ & -3.085 & $(0.061)$ & -0.118 & $(0.043)$ \\
\hline & $2(\mathrm{BED})$ & -7.115 & $(0.162)$ & -1.710 & $(0.055)$ & 3.533 & $(0.148)$ \\
\hline & 3 (MOB) & -5.365 & $(0.096)$ & -0.733 & $(0.045)$ & 5.699 & $(0.267)$ \\
\hline & $4(\mathrm{DRS})$ & -8.042 & $(0.228)$ & -2.230 & $(0.056)$ & 1.999 & $(0.082)$ \\
\hline & 5 (BTH) & -4.457 & $(0.073)$ & -0.205 & $(0.039)$ & 6.159 & $(0.287)$ \\
\hline & 6 (TLT) & -6.638 & $(0.152)$ & -1.735 & $(0.050)$ & 2.457 & $(0.099)$ \\
\hline \multirow{6}{*}{$\beta_{1 * *}$} & 1 (EAT) & 0.362 & $(0.019)$ & 0.345 & $(0.009)$ & 0.098 & $(0.006)$ \\
\hline & $2(\mathrm{BED})$ & 0.396 & $(0.010)$ & 0.540 & $(0.013)$ & 0.286 & $(0.013)$ \\
\hline & 3 (MOB) & 0.347 & $(0.007)$ & 0.510 & $(0.012)$ & 0.419 & $(0.021)$ \\
\hline & 4 (DRS) & 0.394 & $(0.013)$ & 0.454 & $(0.011)$ & 0.199 & $(0.008)$ \\
\hline & $5(\mathrm{BTH})$ & 0.292 & $(0.006)$ & 0.414 & $(0.009)$ & 0.438 & $(0.023)$ \\
\hline & 6 (TLT) & 0.336 & $(0.010)$ & 0.465 & $(0.011)$ & 0.229 & $(0.010)$ \\
\hline \multirow{6}{*}{$A g e_{1 / 2}$} & 1 (EAT) & 104.964 & $(0.492)$ & 88.956 & $(0.174)$ & 81.215 & $(0.483)$ \\
\hline & $2(\mathrm{BED})$ & 97.991 & $(0.176)$ & 83.168 & $(0.092)$ & 67.651 & $(0.184)$ \\
\hline & 3 (MOB) & 95.454 & $(0.147)$ & 81.438 & $(0.086)$ & 66.384 & $(0.155)$ \\
\hline & 4 (DRS) & 100.435 & $(0.242)$ & 84.914 & $(0.109)$ & 69.943 & $(0.204)$ \\
\hline & $5(\mathrm{BTH})$ & 95.241 & $(0.158)$ & 80.495 & $(0.094)$ & 65.939 & $(0.166)$ \\
\hline & 6 (TLT) & 99.784 & $(0.246)$ & 83.731 & $(0.099)$ & 69.287 & $(0.185)$ \\
\hline
\end{tabular}

Table 7: Posterior means for parameters of interest for basic model with $K=3$ extreme profiles. Numbers between parenthesis are posterior standard deviations. 


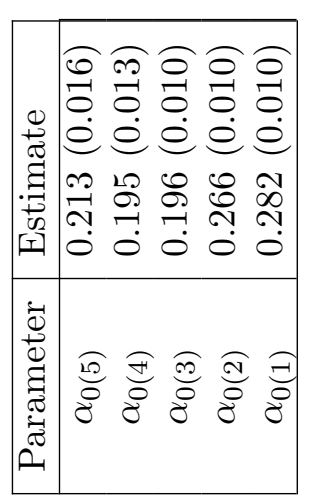

\begin{tabular}{|c|c|c|c|c|c|}
\hline \multirow{3}{*}{ 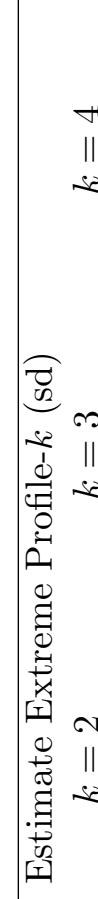 } & 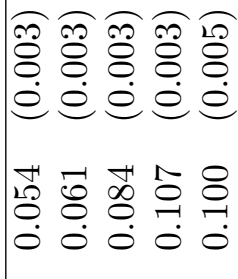 & \multirow{3}{*}{ 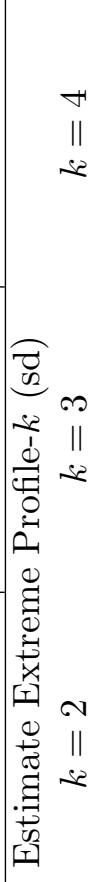 } & 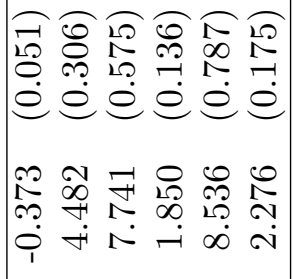 & 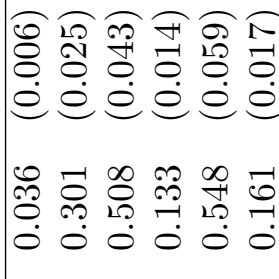 & 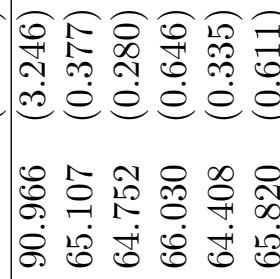 \\
\hline & 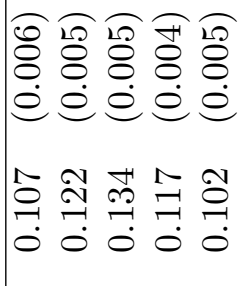 & & 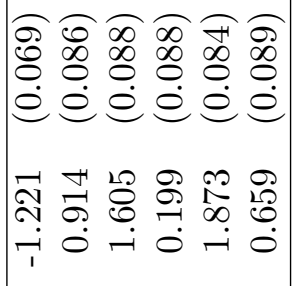 & 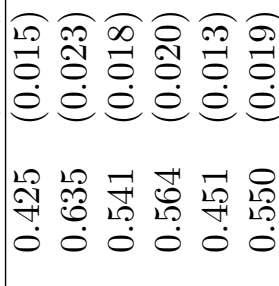 & 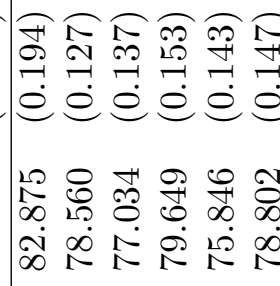 \\
\hline & 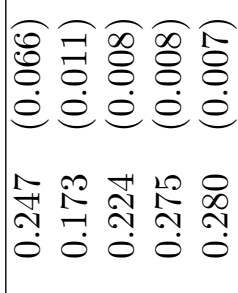 & & 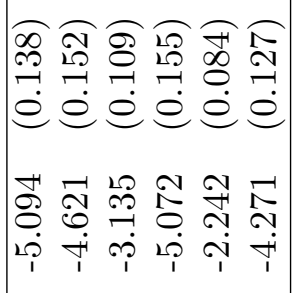 & 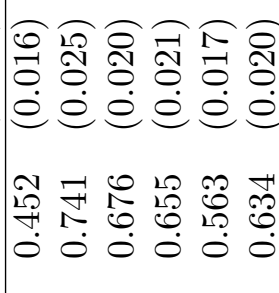 & 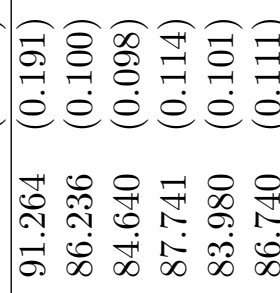 \\
\hline II & 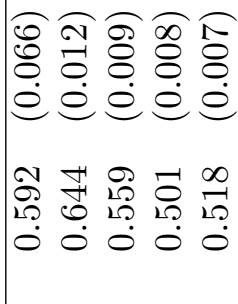 & $\begin{array}{l}71 \\
\| \\
\end{array}$ & 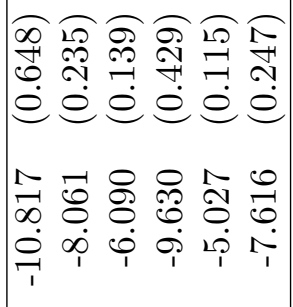 & 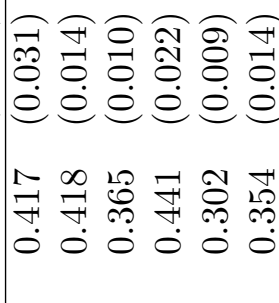 & 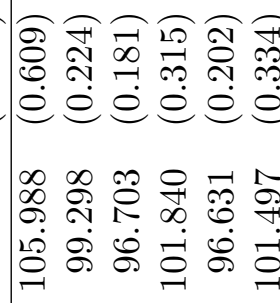 \\
\hline 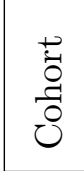 & 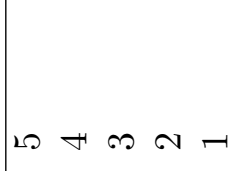 & & 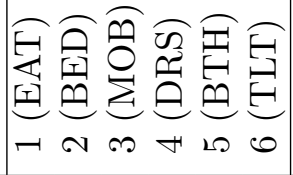 & 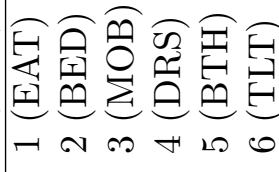 & 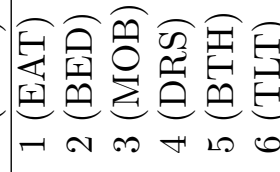 \\
\hline 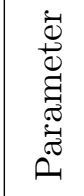 & $u$ & 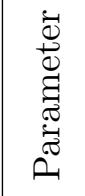 & लं & $\stackrel{2}{*}^{*}$ & $\overrightarrow{\vec{\nabla}}$ \\
\hline
\end{tabular}

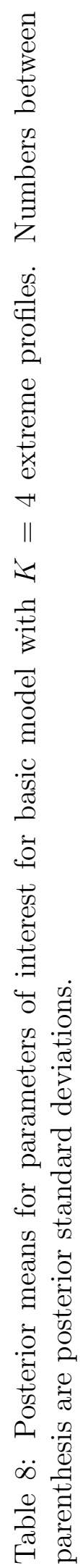




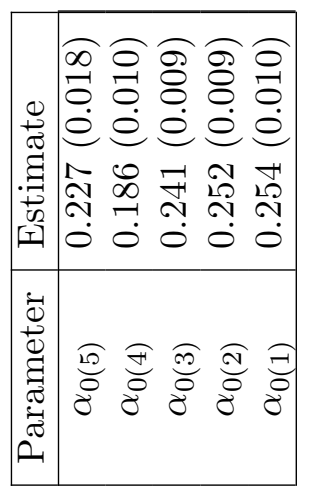

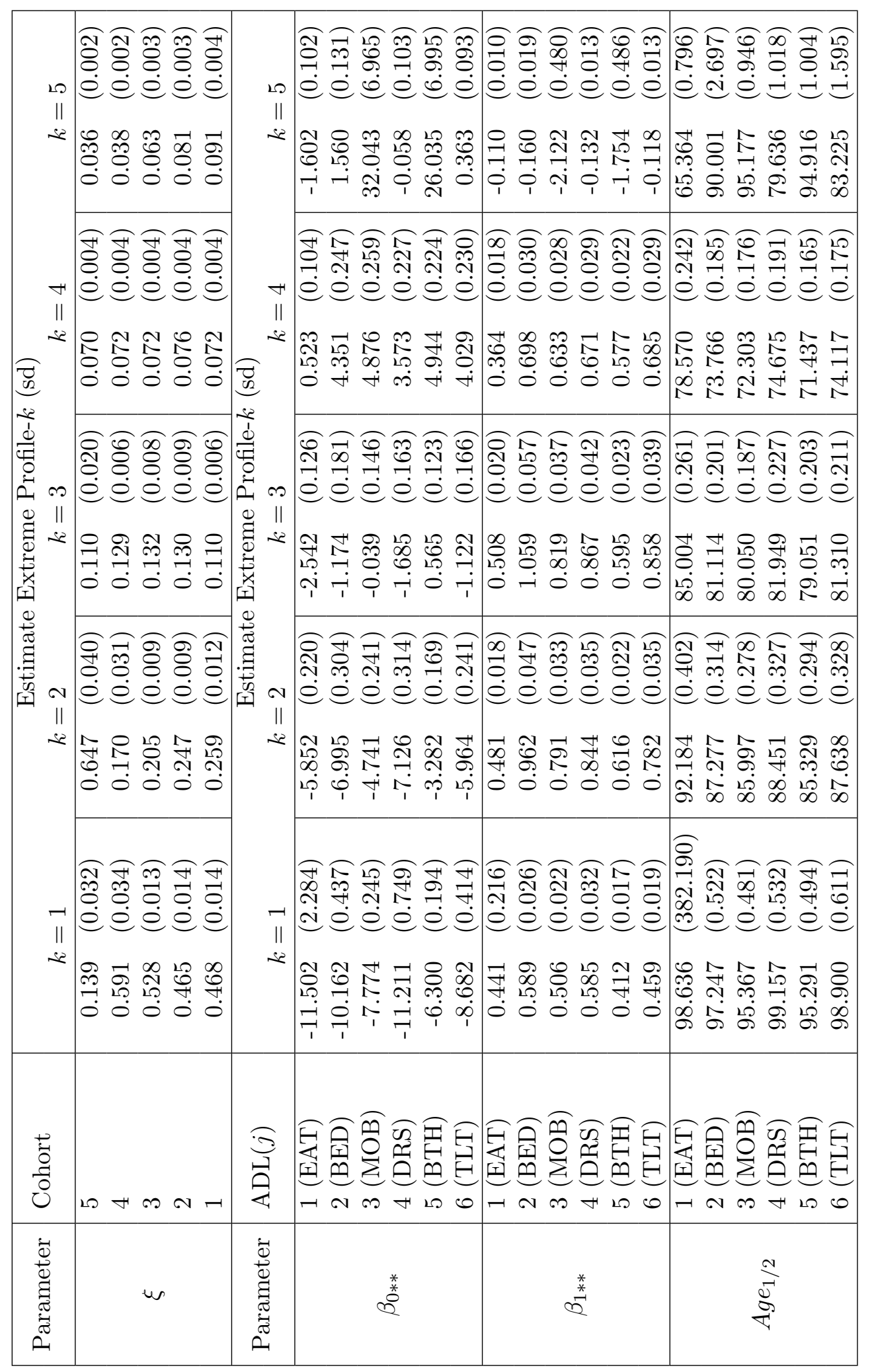

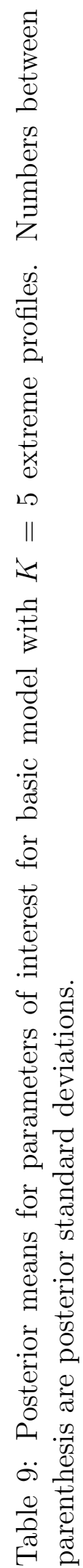

